\title{
Prediction of Radiation-Induced Hypothyroidism Using Radiomic Data Analysis Does Not Show Superiority over Standard Normal Tissue Complication Models
}

\author{
Urszula Smyczynska ${ }^{1,+}{ }^{\oplus}$, Szymon Grabia ${ }^{1,+}{ }^{\circ}$, Zuzanna Nowicka $\left.^{1}{ }^{(}\right)$, Anna Papis-Ubych ${ }^{2}$, Robert Bibik ${ }^{3}$,

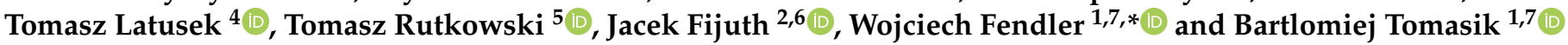 \\ 1 Department of Biostatistics and Translational Medicine, Medical University of Lodz, 92-215 Lodz, Poland; \\ urszula.smyczynska@umed.lodz.pl (U.S.); szymon.grabia@umed.lodz.pl (S.G.); \\ zuzanna.nowicka@umed.lodz.pl (Z.N.); bartlomiej.tomasik@umed.lodz.pl (B.T.) \\ 2 Department of Radiotherapy, N. Copernicus Memorial Regional Specialist Hospital, 93-513 Lodz, Poland; \\ anna_papis@interia.pl (A.P.-U.); jacek.fijuth@umed.lodz.pl (J.F.) \\ 3 Department of Radiation Oncology, Oncology Center of Radom, 26-600 Radom, Poland; rmbibik@wp.pl \\ 4 Radiotherapy Department, Maria Sklodowska-Curie National Research Institute of Oncology \\ (MSCNRIO)—Branch in Gliwice, 44-101 Gliwice, Poland; tomasz.latusek@io.gliwice.pl \\ 5 I Radiation and Clinical Oncology Department, Maria Sklodowska-Curie National Research Institute of \\ Oncology (MSCNRIO)—Branch in Gliwice, 44-101 Gliwice, Poland; Tomasz.Rutkowski@io.gliwice.pl \\ check for \\ updates \\ Citation: Smyczynska, U.; Grabia, \\ 6 Department of Radiotherapy, Chair of Oncology, Medical University of Lodz, 93-509 Lodz, Poland \\ 7 Department of Radiation Oncology, Dana-Farber Cancer Institute, Boston, MA 02115, USA \\ * Correspondence: wojciech_fendler@dfci.harvard.edu \\ + These authors contributed equally to this work.
} S.; Nowicka Z.; Papis-Ubych, A.; Bibik, R.; Latusek, T.; Rutkowski, T.; Fijuth, J.; Fendler, W.; Tomasik, B. Prediction of Radiation-Induced Hypothyroidism Using Radiomic Data Analysis Does Not Show Superiority over Standard Normal Tissue Complication Models. Cancers 2021, 13, 5584. https://doi.org/ $10.3390 /$ cancers 13215584

Academic Editor: Nikolaos Papanikolaou

Received: 23 September 2021 Accepted: 4 November 2021 Published: 8 November 2021

Publisher's Note: MDPI stays neutral with regard to jurisdictional claims in published maps and institutional affiliations.

Copyright: () 2021 by the authors. Licensee MDPI, Basel, Switzerland. This article is an open access article distributed under the terms and conditions of the Creative Commons Attribution (CC BY) license (https:// creativecommons.org/licenses/by/ $4.0 /)$.
Simple Summary: Radiation-induced hypothyroidism (RIHT) commonly develops in cancer survivors that receive radiation therapy for cancers in the head and neck region. The state-of-art normal tissue complication probability (NTCP) models perform satisfactorily; however, they do not use the whole spectrum of information that can be obtained from imaging techniques. The radiomic approach offers the ability to efficiently mine features, which are imperceptible to the human eye, but may provide crucial data about the patient's condition. We gathered CT images and clinical data from 98 patients undergoing radiotherapy for head and neck cancers, 27 of whom later developed RIHT. For them, we created machine-learning models to predict RIHT using automatically extracted radiomic features and appropriate clinical and dosimetric parameters. We also validated the wellestablished external state-of-art NTCP models on our datasets and observed that our radiomic-based models performed very similarly to them. This shows that automated tools may perform as well as the current standard but can be theoretically applied faster and be implemented into existing imaging software used when planning radiotherapy.

Abstract: State-of-art normal tissue complication probability (NTCP) models do not take into account more complex individual anatomical variations, which can be objectively quantitated and compared in radiomic analysis. The goal of this project was development of radiomic NTCP model for radiationinduced hypothyroidism (RIHT) using imaging biomarkers (radiomics). We gathered CT images and clinical data from 98 patients, who underwent intensity-modulated radiation therapy (IMRT) for head and neck cancers with a planned total dose of 70.0 Gy (33-35 fractions). During the 28-month (median) follow-up 27 patients (28\%) developed RIHT. For each patient, we extracted 1316 radiomic features from original and transformed images using manually contoured thyroid masks. Creating models based on clinical, radiomic features or a combination thereof, we considered 3 variants of data preprocessing. Based on their performance metrics (sensitivity, specificity), we picked best models for each variant $((0.8,0.96),(0.9,0.93),(0.9,0.89)$ variant-wise $)$ and compared them with external NTCP models $((0.82,0.88),(0.82,0.88),(0.76,0.91))$. We showed that radiomic-based models did not outperform state-of-art NTCP models $(p>0.05)$. The potential benefit of radiomic-based approach is that it is dose-independent, and models can be used prior to treatment planning allowing faster selection of susceptible population. 
Keywords: radiomics; radiation-induced hypothyroidism; NTCP; head; neck cancer

\section{Introduction}

Patients with head and neck cancer (HNC) treated with radiation therapy (RT) may experience multiple adverse normal tissue effects [1], including hypothyroidism. Radiationinduced hypothyroidism (RIHT) has been reported in $25-65 \%$ of patients and typically develops during the first 2 years from RT completion. Since the symptoms of RIHT are non-specific and insufficient levels of thyroid hormones not only negatively impact patients quality of life [2], but also mortality [3,4], adequate identification of patients at risk of this effect is of paramount importance [5-7].

Clinical and dosimetric parameters are typically used in normal tissue complication probability (NTCP) models to predict RIHT [1]. Recently, we [7] and others [6] have applied published NTCP models in homogenous cohorts of patients with oropharyngeal cancer (OPC). We concluded that two models based on thyroid mean dose and volume, published by Rønjom et al. [8] and by Boomsma et al. [9], performed best in terms of accuracy (84 to $87 \%$ ), highlighting the feasibility of dose-response models to predict RIHT and their potential utility in the clinical setting.

Radiomics is a relatively new discipline that aims at deriving biomarkers from medical images [10]. These biomarkers are features describing shape, intensity or texture of specific region(s) of interest (ROI), typical an organ or lesion. Radiomics emerged in the field of cancer studies where large number of medical images are generated even by routine diagnostic process. In this context, radiomics can supplement standard radiologic assessment with localized, quantitative information on interesting structures with low cost and without any additional burden or discomfort to patients. In recent years, radiomics researchers community took considerable efforts to standardize methodology aiming at translatability of radiomic studies that resulted in publication of the first reference manual for image biomarker studies [11].

Radiomic features were shown to reflect cancer biology in terms of histologic type [12], overall and progression-free survival [12-14], probability of recurrence [15], activity of biological pathways [12,16], human papillomavirus infection [17], probability lymph node [18] or distant metastases [19], CD8 cells infiltration [20]. Some studies extended radiomic analysis outside tumor volume, including also peritumoral regions for prediction of patients survival [21] or adjuvant chemotherapy [22]. Similarly, lymph nodes were sometimes considered to be ROI in studies that aimed at identifying lymph nodes metastases [23,24]. Some radiomic NTCP models based on computed tomography (CT) were developed, for instance for prediction of xerostomia after head and neck cancer radiotherapy [25,26], radiation-induced pneumonitis [27]. Alternatively, radiomic features for radiation-induced NTCP models could be calculated in 3D dose distribution that was successfully done for cervical cancer [28], prostate cancer [29] and lung cancer [30]. Given the very good performance of some NTCP models in predicting RIHT in patients with OPC, the question whether there would be an added benefit of an in depth radiomic analysis was unresolved and thus we decided to apply CT-based radiomics in this group of patients and compare predictive performance with the available models based on dosimetric and clinical features.

\section{Materials and Methods}

\subsection{Patients and CT Images}

The studied cohort was a subgroup of patients included in previous study on validation of NTCP models for radiation-induced hypothyroidism by Nowicka et al. [7], recruited between 1 May 2016 and 31 December 2018 and followed up until February 2020. In addition to already collected clinical data, for this study we retrieved CT images from radiotherapy planning with thyroid glands retrospectively contoured by two experienced radiation oncologists according to the guidelines for organs at risk (OARs), as described 
previously. Patients for whom the CT image was unavailable, thyroid contour was missing or thyroid region contained CT artifacts, were excluded. Finally, among 108 patients recruited in 3 centers 98 had complete data and were included in this study (38 from center A-Copernicus Regional Specialist Hospital in Lodz, Poland; 12 from center B-Radom Oncology Center and Maria Sklodowska-Curie National Research Institute of Oncology, Radom, Poland and 48 from center C-Radiotherapy Department, Maria Sklodowska-Curie National Research Institute of Oncology — branch in Gliwice, Poland); selection of cases is summarized in Figure 1A.

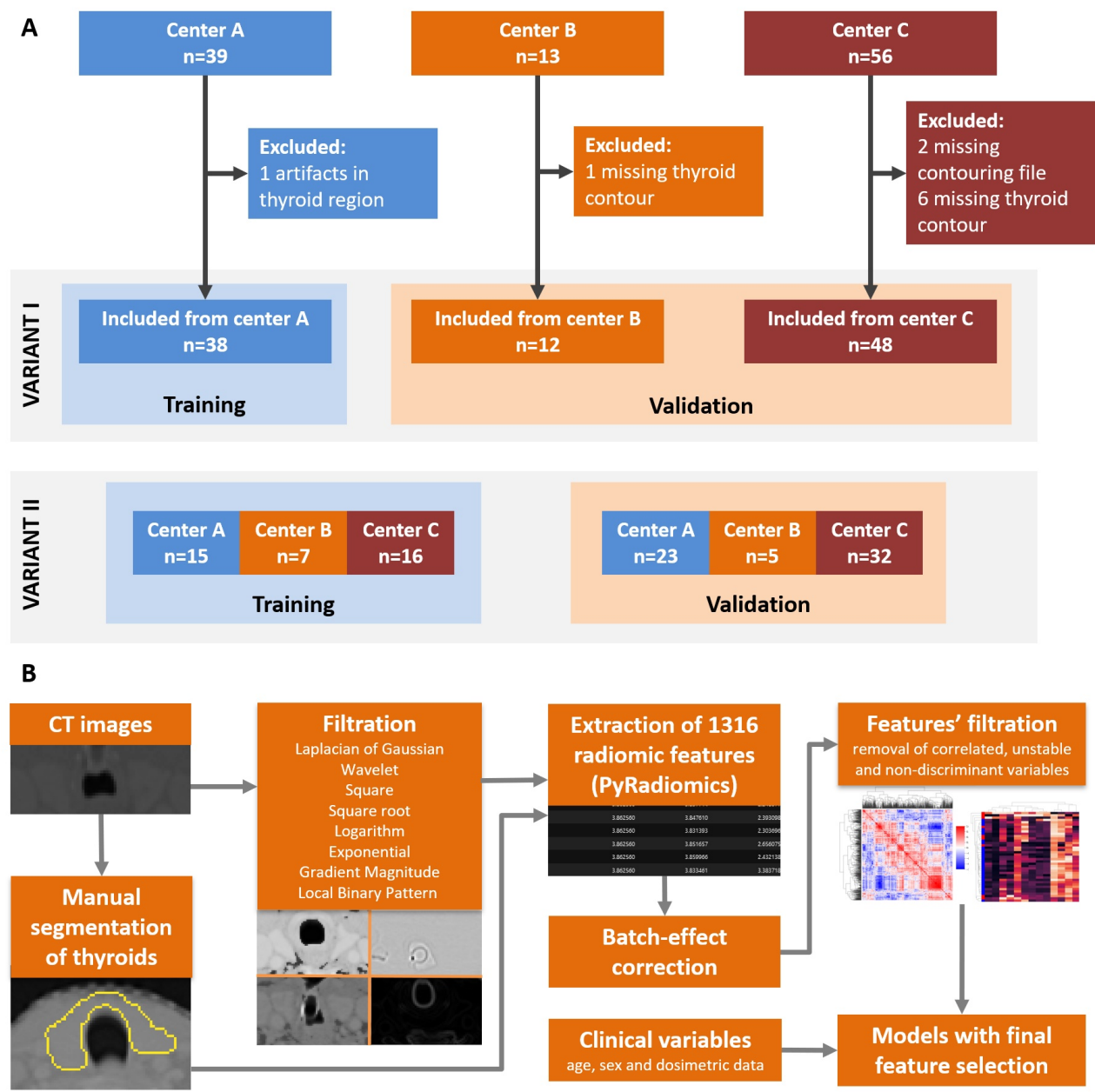

Figure 1. Summary of patients' selection and data subsets (A). Outline of the study from image segmentation to NTPC model derivation (B).

All patients underwent intensity-modulated radiation therapy (IMRT) of OPC before which their thyroid function was normal. After RT, they were monitored for a median of 28 months on average (median). During this follow-up period, 27 patients developed hypothyroidism. To avoid bias, laboratory assessment, contouring, outcome assessments, statistical and radiomic analysis were performed by independent researchers. Details of treatment protocol were described previously [7].

For all 98 patients, CT images paired with RT plans and anatomical structure contouring files were retrieved from PACS of treating centers. All images were stored in DICOM format in CT and RTSTRUCT modalities; however, some differences in acquisition protocol were identified. First, each center used a different CT scanner (center A: Somatom Sensation Open, Siemens; center B: Optima CT580 RT, GE Healthcare; center C: SOMATOM Definition AS and Somatom Sensation Open, Siemens). In each center, slice thickness and pixel spacing were selected by radiotherapist and radiologist so that images were sufficient 
for treatment planning. Summary of selected settings in each center is presented in Table 1 and anonymized raw patients data are in Table S1 in Supplementary Materials.

The study was approved by the Bioethics Committee of the Medical University of Lodz (KE/7/10, RNN/65/18).

Table 1. Patient, disease and CT images characteristics.

\begin{tabular}{|c|c|c|c|c|c|c|c|}
\hline \multirow[b]{2}{*}{ RIHT } & & \multicolumn{2}{|c|}{ Center A $(n=38)$} & \multicolumn{2}{|c|}{ Center $B(n=12)$} & \multicolumn{2}{|c|}{ Center $C(n=48)$} \\
\hline & & $\mathrm{NO}$ & YES & $\mathrm{NO}$ & YES & NO & YES \\
\hline \multirow[t]{2}{*}{ Sex } & Female & 7 & 7 & 1 & 1 & 5 & 2 \\
\hline & Male & 21 & 3 & 8 & 2 & 29 & 12 \\
\hline \multirow[t]{2}{*}{ Age } & Median & 62.0 & 60.0 & 61.0 & 57.0 & 57.5 & 58.0 \\
\hline & IQR & $57.0-66.2$ & $56.8-61.8$ & $60.0-68.0$ & $55.5-60.5$ & $53.0-62.0$ & $52.2-66.5$ \\
\hline \multirow[t]{2}{*}{ Stage } & $\widehat{\mathrm{I}-\mathrm{II}}$ & 6 & 1 & 2 & 0 & 12 & 1 \\
\hline & III-IV & 22 & 9 & 7 & 3 & 22 & 13 \\
\hline \multirow{2}{*}{ Mean thyroid dose, $D_{\text {mean }}(\mathrm{Gy})$} & Median & 54.8 & 57 & 55.2 & 57.3 & 47.2 & 52.5 \\
\hline & IQR & $51.9-56.3$ & $52.7-59.3$ & $53.5-56.3$ & $56.2-58.4$ & $43.8-49.5$ & $49.7-56.2$ \\
\hline \multirow{2}{*}{ Minimal thyroid dose, $D_{\min }(\mathrm{Gy})$} & Median & 42.5 & 46.5 & 43 & 51.8 & 30.9 & 44.3 \\
\hline & IQR & $29.1-46.6$ & $43.3-47.5$ & $41.1-48.2$ & $50.1-54.3$ & $24.6-39.0$ & $32.7-46.9$ \\
\hline \multirow{2}{*}{ Median thyroid dose, D50 (Gy) } & Median & 55.0 & 55.5 & 54.5 & 58.5 & 47.0 & 52.2 \\
\hline & IQR & $53.7-56.3$ & $52.9-58.7$ & $53.9-54.9$ & $57.2-59.0$ & $43.8-50.4$ & $50.5-56.4$ \\
\hline \multirow{2}{*}{ Maximal thyroid dose, $D_{\max }(\mathrm{Gy})$} & Median & 62.5 & 69.4 & 61.7 & 61.6 & 60.2 & 65.1 \\
\hline & IQR & $57.6-70.1$ & $63.3-71.9$ & $60.8-62.1$ & $59.6-61.8$ & $52.6-68.0$ & $53.6-72.2$ \\
\hline \multirow{2}{*}{ Thyroid volume (ml) } & Median & 21.7 & 11.8 & 29 & 12.6 & 19.1 & 10.6 \\
\hline & IQR & $19.0-32.9$ & $7.7-13.9$ & $21.7-37.4$ & $10.6-14.0$ & $14.6-27.6$ & $8.3-13.3$ \\
\hline \multirow{2}{*}{ Baseline fT4 (pg/mL) } & Median & 6.5 & 6.1 & 9.3 & 8.2 & 7.2 & 8.1 \\
\hline & IQR & $5.3-7.4$ & $5.1-7.6$ & $8.0-10.1$ & 7.7-10.7 & $6.3-8.4$ & 7.9-9.9 \\
\hline \multirow{2}{*}{ Baseline TSH (mIU/L) } & Median & 0.5 & 1.3 & 0.7 & 0.4 & 0.7 & 1.1 \\
\hline & IQR & $0.3-0.8$ & $0.8-1.7$ & $0.6-1.2$ & $0.4-0.7$ & $0.5-1.5$ & $0.6-1.2$ \\
\hline \multirow{2}{*}{ Mean pituitary dose (Gy) } & Median & 4.0 & 3.8 & 4.0 & 3.8 & 3.8 & 3.7 \\
\hline & IQR & $3.0-4.5$ & $3.0-5.3$ & $3.2-4.8$ & $3.6-3.8$ & $3.0-4.4$ & $3.0-4.8$ \\
\hline \multirow{2}{*}{ Time to follow-up (months) } & Median & 29.5 & 15 & 22 & 13 & 38 & 19 \\
\hline & IQR & $26.0-37.2$ & $14.0-15.8$ & $21.0-24.0$ & $12.0-13.5$ & $31.2-41.0$ & $16.0-21.0$ \\
\hline \multirow{8}{*}{ Pixel spacing $\left(\mathrm{mm}^{2}\right)$} & $0.98 \times 0.98$ & 25 & 9 & 0 & 0 & 26 & 11 \\
\hline & $1.07 \times 1.07$ & 1 & 0 & 0 & 0 & 3 & 3 \\
\hline & $1.09 \times 1.09$ & 0 & 0 & 0 & 0 & 1 & 0 \\
\hline & $1.11 \times 1.11$ & 0 & 0 & 0 & 0 & 1 & 0 \\
\hline & $1.13 \times 1.13$ & 0 & 0 & 0 & 0 & 1 & 0 \\
\hline & $1.17 \times 1.17$ & 0 & 0 & 0 & 0 & 1 & 0 \\
\hline & $1.27 \times 1.27$ & 1 & 0 & 9 & 3 & 1 & 0 \\
\hline & $1.56 \times 1.56$ & 1 & 1 & 0 & 0 & 0 & 0 \\
\hline \multirow{6}{*}{ Slice thickness (mm) } & 1.5 & 1 & 0 & 0 & 0 & 0 & 0 \\
\hline & 2 & 1 & 1 & 0 & 0 & 2 & 1 \\
\hline & 2.5 & 0 & 0 & 9 & 3 & 0 & 0 \\
\hline & 3 & 24 & 9 & 0 & 0 & 26 & 9 \\
\hline & 4 & 0 & 0 & 0 & 0 & 6 & 4 \\
\hline & 5 & 2 & 0 & 0 & 0 & 0 & 0 \\
\hline
\end{tabular}

\subsection{Image Preprocessing and Radiomic Features Calculation}

Image processing and radiomic feature extraction were performed with PyRadiomics v3.0 [31]. Due to diversity of pixel spacing and slice thicknesses, all images and thyroid masks (generated from contours using dcmrtstruct2nii library [32]) were resampled to $1 \times 1 \times 1 \mathrm{~mm}^{3}$ isotropic voxels. Default PyRadiomics interpolators were used in resampling: nearest neighbor interpolation for binary thyroid mask and B-spline interpolation for CT image. At the same time, both image and mask were cropped to thyroid bounding box with $10 \mathrm{~mm}$ padding added to ROI bounding box at each side; the operation is done by default by PyRadiomics during resampling, but we increased default padding size from 5 to 10 voxels. Then, radiomic features were calculated that in the applied version of the library included: 14 shape features, 18 first order statistics, 24 gray level cooccurrence matrix-based 
(GLCM) features, 16 gray level run length matrix-based (GLRLM) features, 16 gray level size zone matrix-based (GLSZM) features, 5 neighborhood gray tone difference matrixbased (NGTDM) features, 14 gray level dependence matrix-based (GLDM) features (full list of features in Appendix A). Radiomic features were extracted from original images and from their filtered versions, applying all filters available in PyRadiomics feature extractor: square, square root, logarithm, gradient, exponential and 8 wavelet decomposition filters. Default values of PyRadiomics feature extractor settings were used, including filtration parameters. Resegmentation was not applied. In total, 1316 features were calculated for each image (14 shapes features extracted from image mask, 93 intensity-based features for original images and 13 filtered ones).

The processing of images and NTCP model derivation is summarized in Figure 1B, while raw radiomic features values are reported in Table S2.

\subsection{Stability Assessment}

The purpose of stability analysis was to identify features that were unaffected (or affected only slightly) by minor differences in contouring of thyroid glands. As the segmentations by multiple radiotherapists or radiologists were not available, we decided to perform a simulation study. We modelled inaccuracies of contouring as affine deformations of thyroid mask, consisting of:

- 3 translations by up to $1 \mathrm{~mm}$ in either direction along each of the 3 main axes,

- 3 rotations by up to $2^{\circ}$ in either direction around each of the 3 main axes,

- 3 zooms by up to $2 \%$ of either dimension along each of the 3 main axes.

We generated 100 such transformations by randomly choosing order and parameters of the above 9 operations. Then, we applied every transformation to all thyroid masks in our image data set and used the transformed masks to calculate new sets of radiomic features. These transformed masks were used solely for the purpose of stability assessment, while for model development we calculated features using original manual contours of thyroid glands.

Inter-class correlation coefficient (ICC) served as a measure of agreement between original features (calculated using unchanged mask) and those calculated using each of 100 transformed masks. Later, 100 ICC values obtained for different transformed masks were averaged to give single measure of stability for each feature. Stability was classified as excellent when mean ICC exceeded 0.9, good (ICC between 0.75 and 0.9 ), moderate (ICC between 0.5 and 0.75 ) or poor (ICC not exceeding 0.5 ).

\subsection{Feature Preprocessing and Splitting Data Set}

Raw feature values were first scaled to a $[-1,1]$ range, then subjected to Yeo-Johnson transformation [33] and scaled to [-1,1] range once again to facilitate machine-learning model derivation. First scaling was performed to avoid exponentiation (included in YeoJohnson transformation) of raw radiomic features with high absolute values, such as energy that easily reached values at the order of $10^{10}$. Second scaling aimed at facilitating model training by limiting the range of values generated by Yeo-Johnson transformation. As average values and distributions of features values varied across the samples (Figure S1) we normalized them dividing each value by sample mean. While performing normalization, we followed the reasoning common for other high-throughput studies (transcriptomics, proteomics) that usually only minority of features should be expected to differ between conditions, thus the distribution of all feature values should not differ significantly between samples.

We observed that even after normalization, we could still observe batch effect related to clinics that performed CT scans (Figure S2). Thus, to account for this and check the impact of batch on NTCP model performance, we decided to implement different variants of data preprocessing and splitting:

- Variant I without batch effect correction, later referred to as Variant Ia: center A as training set, centers B and $C$ as validation set as shown in Figure 1A. 
- Variant I with batch effect removed by ComBat [34], referred to also as Variant $\mathrm{Ib}$ : center $A$ as training set, centers $B$ and $C$ as validation set.

- Variant II: no batch effect removal, but data from three centers were joined and subsequently divided into training and validation sets (Figure 1A). Training set contained 38 patients including 10 RITH to match the number of patients and proportion of RITH cases of center A data set so that both variants of splitting data are as comparable as possible .

\subsection{Feature Filtration}

Due to the large number of features, we decided to filter them before derivation of NTCP models. First, only features with excellent or good stability were considered to be candidates for NTCP model predictors. We used ICC classes from center A for filtration in Variant I and, analogously ICC classes from training set for Variant II.

Then, to retain only features that actually differentiate patient with and without RIHT in follow-up, t-tests were performed to compare values of each stable feature between these group. Benjamini-Hochberg correction was applied to $\mathrm{p}$-values to control false discovery rate (FDR) in this large set of comparisons. Next, hierarchical clustering of features was performed with $1-\mathrm{r}$ (correlation coefficient) as distance measure and average linkage to identify groups of highly correlated features. These groups of features were extracted by setting a threshold of 0.3 to distances in the dendrogram. From each such group we retained a single feature with lowest FDR-corrected p-value (later referred to as FDR) on the condition that this FDR did not exceed 0.1. Additionally, we kept all features with FDR $<0.01$ even if they were correlated with others. This shortened list of features was the basis for models' training that included final model-based feature selection.

\subsection{Model Training and Evaluation}

Model training was performed with the use of scikit-learn Python library [35]. Model architectures considered in our analysis included: logistic regression, multilayer perceptron (MLP), k nearest neighbors classifier, support vector classifier, decision tree, random forest, AdaBoost classifier, Gaussian process classifier, Gaussian Naive Bayes classifier, Quadratic discriminant analysis. Whenever possible (in logistic regression, support vector classifiers, decision trees and random forest) two methods of weighting cases during training were applied: (1) equal weights of all cases (2) weights inversely proportional to classes' frequencies to account for imbalanced data set with less than $1 / 3$ of cases in RIHT group. Full list of tested model parameters is collected in Table S3.

Models were derived using three feature sets: (1) only clinical and dosimetric features (clinical model), (2) only radiomic features (radiomic model), (3) radiomic, clinical and dosimetric features (radiomic+clinical model). Final set of input features for each model was determined by forward method of Sequential Feature Selector from mlxtend library [36]. Feature selector used area under the ROC curve (AUC) as a measure of models' performance and was allowed to choose from 2 to 5 features.

All models were first derived using training data and then their performance was tested on validation data. AUC, accuracy, sensitivity, specificity and F-score were calculated for each model. The analysis was performed for Variants Ia, Ib and II of clinical and radiomic features derivation. Models with the highest F-score were selected for each variant of analysis and input feature set. Finally, ensembles of best clinical and radiomic models were considered that combined them in following ways:

- logical conjunction (AND): positive prediction only when both models predicted RIHT,

- logical disjunction (OR): positive prediction when any of the two models predicted RIHT,

- $\quad$ averaged probability (PROBA): probability (raw output) of two models were averaged and new decision threshold selected using ROC curve for training set. 


\section{Results}

\subsection{Feature Stability Analysis}

Results of feature stability analysis differed slightly between variant I and II. In variant $\mathrm{I}$, where all images were acquired in the same center, we identified more features as highly stable (ICC > 0.9) than in variant II (721, Figure 2A vs. 621 features, Figure 2B). In line with this observation, lowest stability class was assigned to 211 in variant II and only to 138 features in variant I (stability analysis was performed before batch correction, thus at this stage variant $\mathrm{Ia}$ and $\mathrm{Ib}$ are not distinguished). Independently of the variant, all shape features were excellently stable even though our test consisted essentially in disturbing thyroid mask shape. Excellent or good stability was observed for majority of first order, GLCM, GLRLM, and GLDM features, while for GLSZM and NGTDM the fraction of unstable features was higher. In majority, stable features overlapped between variants I and II (Figure 2C). Stability of features depended also on the applied filter (Figure 2D), with exponential and gradient filter ensuring on average higher stability than square, square root, logarithm and some wavelet filters.

In further analysis, we included all features with excellent and good stability: 926 in variant I and 869 in variant II. Stability classes for all features are reported in Table S4.

\begin{tabular}{lrrrr} 
A & \multicolumn{4}{c}{ Stability category in variant I } \\
\cline { 2 - 5 } feature group & excellent & good & moderate & low \\
\hline shape & 14 & 0 & 0 & 0 \\
first order & 141 & 37 & 53 & 21 \\
GLCM & 195 & 60 & 60 & 21 \\
GLRLM & 139 & 23 & 36 & 26 \\
GLSZM & 94 & 47 & 53 & 30 \\
GLDM & 116 & 19 & 37 & 24 \\
NGTDM & 22 & 19 & 13 & 16 \\
total & 721 & 205 & 252 & 138 \\
\hline
\end{tabular}

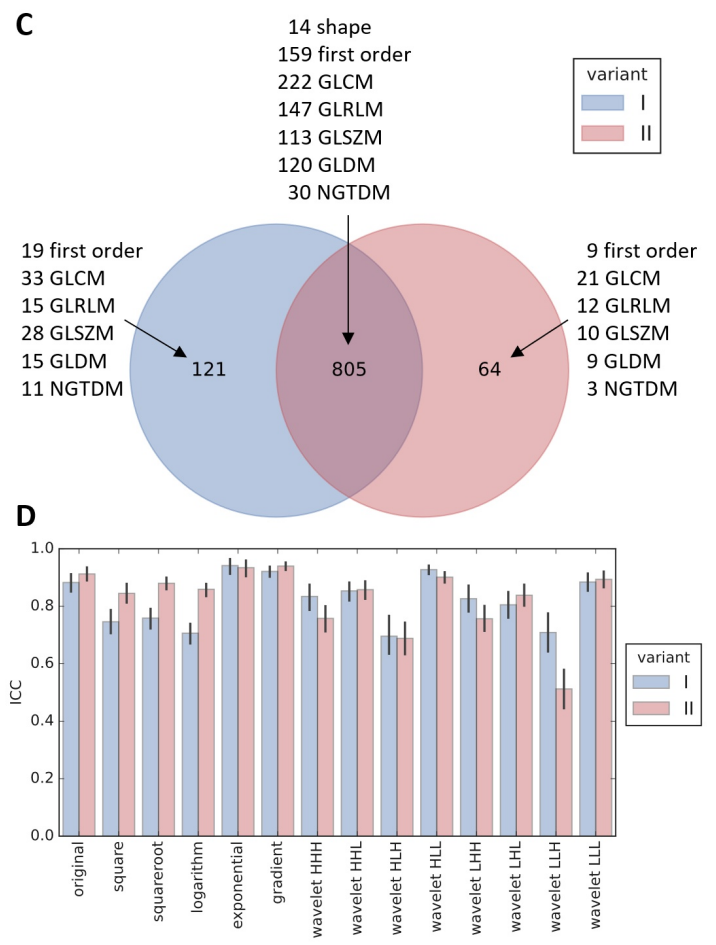

\begin{tabular}{lrrrr}
\hline \multicolumn{5}{c}{ Stability category in variant II } \\
feature group & excellent & good & moderate & low \\
\hline shape & 14 & 0 & 0 & 0 \\
first order & 121 & 47 & 46 & 38 \\
GLCM & 161 & 82 & 46 & 47 \\
GLRLM & 128 & 31 & 36 & 29 \\
GLSZM & 74 & 49 & 55 & 46 \\
GLDM & 103 & 26 & 38 & 29 \\
NGTDM & 20 & 13 & 15 & 22 \\
total & 621 & 248 & 236 & 211 \\
\hline
\end{tabular}

Figure 2. Results of feature stability assessment. Number of features in each group classified to different stability categories for variant I (A) and II (B). Overlap of features with at least good stability between variant I and II (C). Average stability of all radiomic features with respect to applied image filter (D, shown mean with $95 \%$ confidence interval).

\subsection{Feature Processing and Filtration}

Before filtration, features were scaled, normalized (Figure S1) and in variant Ib batch effect was corrected (Figure S2). First stage of filtration consisted of exclusion of features that in univariate analysis did not differentiate patients who did or did not develop RIHT (detailed results of this analysis in Table S5). It resulted in selection of 165 radiomic features in variant I and 166 in variant II, among which 153 were common to both variants (Figure 3A). Having a limited number of patients, we decided to reduce these numbers further before model development by elimination of highly correlated features. Selection of representatives from each group of such related variables left 67 features in variant Ia, 68 
in variant Ib and 66 in variant II (lists of features in Table S6). Again, the overlap between variants, consisting of 61 radiomic features, greatly outnumbered distinct variables for each variant (Figure 3C). At both stages of filtration, features from original image and the one with exponential filter applied were preferred over other groups (Figure 3B,D).

A

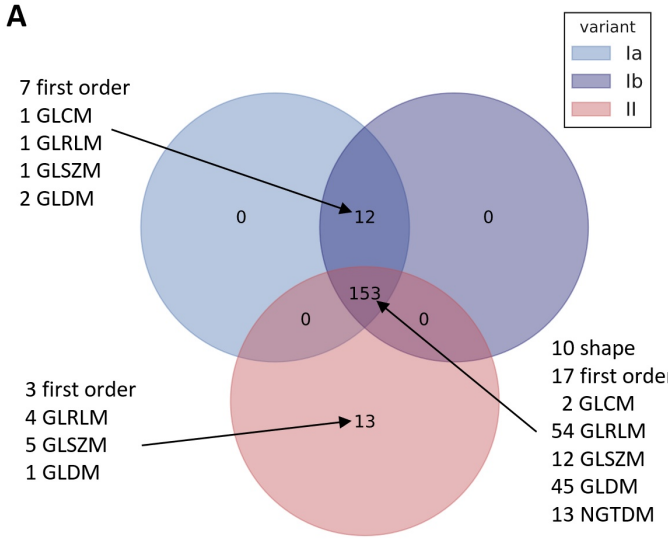

B

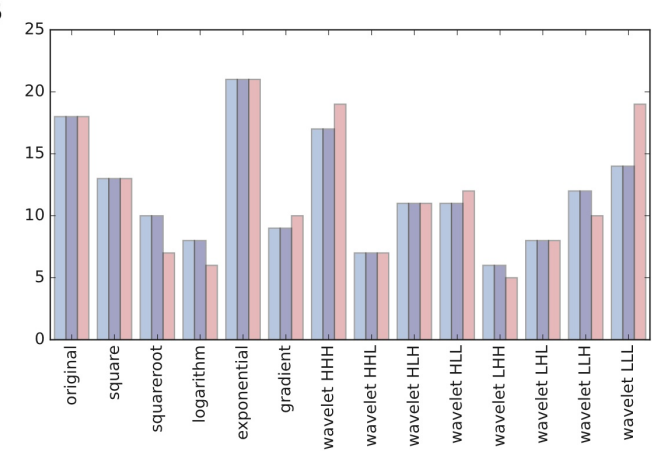

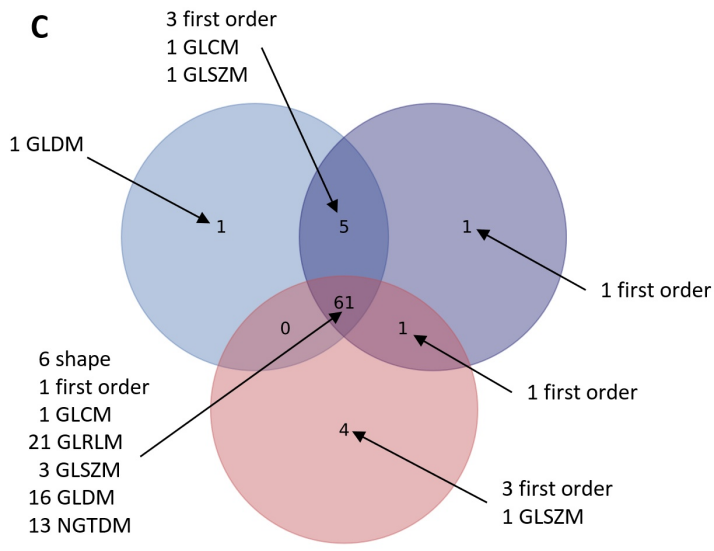

D

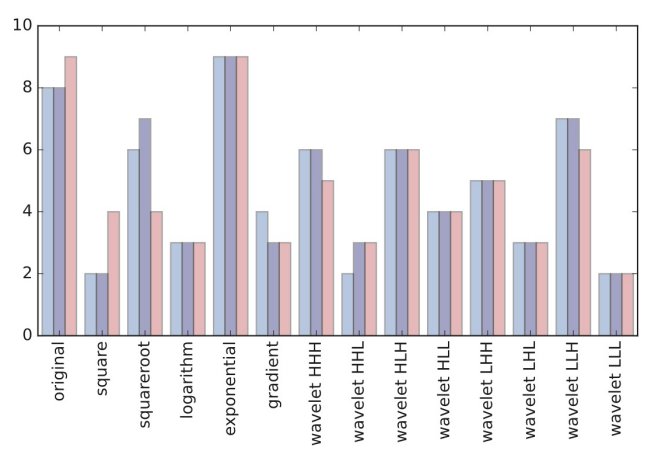

Figure 3. Features with FDR $<0.1$ in univariate analysis grouped by radiomics category (A) and by image filter (B). Features selected for model grouped by radiomics category (C) and by image filter (D).

\subsection{Models}

The training of all the considered models on the reduced set of features and their validation resulted in selection of top 9 models, one for each variant and a feature set. Their names, along with the features they were built upon, are presented in Table 2, while details of all analyzed models can be found in Table S7.

For all the variants, when considering models derived only with clinical features, Gaussian process classifier was chosen. Likewise, the same set of features was selected, i.e., mean of thyroid dose, median of thyroid dose and volume of the thyroid. For radiomic and clinical+radiomic feature sets, the selected models were logistic regression without regularization, with equal class weights and excluded intercept for variant Ia and multilayer perceptron with 4 and 2 hidden neurons for variants $\mathrm{Ib}$ and II, respectively. For those feature sets, selected features were more diverse.

In all variants of analysis, radiomic models performed similarly to clinical models (Figure 4A-C); comparisons of ROC AUC by DeLong test never showed superiority of models using radiomic features vs clinical/dosimetric model (Table 3). Slight improvement of radiomic models is observed after batch effect correction (variant $\mathrm{Ib}$ ). The statistical measures (sensitivity, specificity, accuracy and f-score) for each of the selected models and the ensembles of clinical and radiomic models were calculated (Figure 4D-I). Radiomic+clinical model was not included in ensembles, because it contained a very similar feature set to radiomic and clinical models taken together. Based on those measures, we picked clinical, radiomic and OR models for the comparison with the external NTCP models for radiation-induced hypothyroidism [8,9,37-39]. 
Table 2. Model architectures and features selected for each of the variants and feature sets.

\begin{tabular}{|c|c|c|c|c|c|c|}
\hline & $\begin{array}{l}\text { VARIANT Ia } \\
\text { Model }\end{array}$ & Features & $\begin{array}{l}\text { VARIANT Ib } \\
\text { Model }\end{array}$ & Features & $\begin{array}{l}\text { VARIANT II } \\
\text { Model }\end{array}$ & Features \\
\hline $\begin{array}{l}\text { clinical } \\
\text { (same for Ia and Ib) }\end{array}$ & GPC & $\begin{array}{l}\mathrm{D}_{\text {mean }} \\
\text { D50 } \\
\mathrm{V}_{\text {thyroid }}\end{array}$ & GPC & $\begin{array}{l}\mathrm{D}_{\text {mean }} \\
\text { D50 } \\
\mathrm{V}_{\text {thyroid }}\end{array}$ & GPC & $\begin{array}{l}\mathrm{D}_{\text {mean }} \\
\text { D50 } \\
\mathrm{V}_{\text {thyroid }}\end{array}$ \\
\hline radiomic & $\mathrm{LR}_{\mathrm{E}}$ & $\begin{array}{l}\text { wavelet HHH GLSZM } \\
\text { zone percentage } \\
\text { logarithm NGTDM } \\
\text { coarseness }\end{array}$ & $\mathrm{MLP}_{4}$ & $\begin{array}{l}\text { original NGTDM } \\
\text { coarseness } \\
\text { wavelet LLL NGTDM } \\
\text { coarseness } \\
\text { exponential GLDM } \\
\text { small dependence } \\
\text { low gray level emphasis } \\
\text { logarithm NGTDM } \\
\text { coarseness }\end{array}$ & $\mathrm{MLP}_{4}$ & $\begin{array}{l}\text { exponential GLDM } \\
\text { small dependence } \\
\text { low gray level emphasis } \\
\text { logarithm NGTDM } \\
\text { coarseness }\end{array}$ \\
\hline clinical+radiomic & $\mathrm{MLP}_{2}$ & $\begin{array}{l}\text { sex } \\
\text { original shape } \\
\text { least axis length } \\
\text { exponential GLRLM } \\
\text { run percentage } \\
\text { exponential GLDM } \\
\text { small dependence } \\
\text { low gray level emphasis } \\
\text { logarithm NGTDM } \\
\text { coarseness }\end{array}$ & $\mathrm{MLP}_{4}$ & $\begin{array}{l}\text { original NGTDM } \\
\text { coarseness } \\
\text { wavelet LLL NGTDM } \\
\text { coarseness } \\
\text { exponential GLDM } \\
\text { small dependence } \\
\text { low gray level emphasis } \\
\text { logarithm NGTDM } \\
\text { coarseness }\end{array}$ & $\mathrm{MLP}_{2}$ & $\begin{array}{l}\text { sex } \\
\text { original shape } \\
\text { least axis length } \\
\text { exponential GLRLM } \\
\text { run percentage } \\
\text { exponential GLDM } \\
\text { small dependence } \\
\text { low gray level emphasis } \\
\text { logarithm NGTDM } \\
\text { coarseness }\end{array}$ \\
\hline
\end{tabular}


Table 3. Comparison between best models with and without radiomic features.

\begin{tabular}{llrlrlrr}
\hline & VARIANT Ia & \multicolumn{2}{l}{ VARIANT Ib } & \multicolumn{2}{c}{ VARIANT II } \\
\hline Model & AUC \pm SE & $p$ & AUC \pm SE & $p$ & AUC \pm SE & $p$ \\
clinical & $0.90 \pm 0.07$ & - & $0.90 \pm 0.07$ & - & $0.95 \pm 0.05$ & - \\
radiomic & $0.89 \pm 0.07$ & 0.9196 & $0.94 \pm 0.05$ & 0.6471 & $0.91 \pm 0.07$ & 0.6263 \\
radiomic + clinical & $0.95 \pm 0.05$ & 0.5549 & $0.94 \pm 0.05$ & 0.6471 & $0.92 \pm 0.06$ & 0.8286 \\
PROBA & $0.90 \pm 0.07$ & 1.0000 & $0.95 \pm 0.05$ & 0.5549 & $0.93 \pm 0.06$ & 0.7940 \\
\hline$p$
\end{tabular}

p-values for comparison of each model with radiomic features vs clinical model.

Compared with previous examples (Figure 5), our models tend to be slightly less sensitive, but more specific and accurate. Models by Cella et al. and Vogelious et al. significantly overestimate risk of RIHT, declaring all or almost all patients as having high risk of this complication. The model by Ronjom et al. seemed comparable to our models.

Radiomic features included in the final models came from original, logarithm, exponential and wavelet (LLL, HHH) images. Majority of these features measured nonuniformity of the thyroid region (e.g., coarseness, zone percentage) and were higher in patients who developed RIHT (Figure 6). The only feature lower in these patients was least axis length of thyroid (Figure S3).
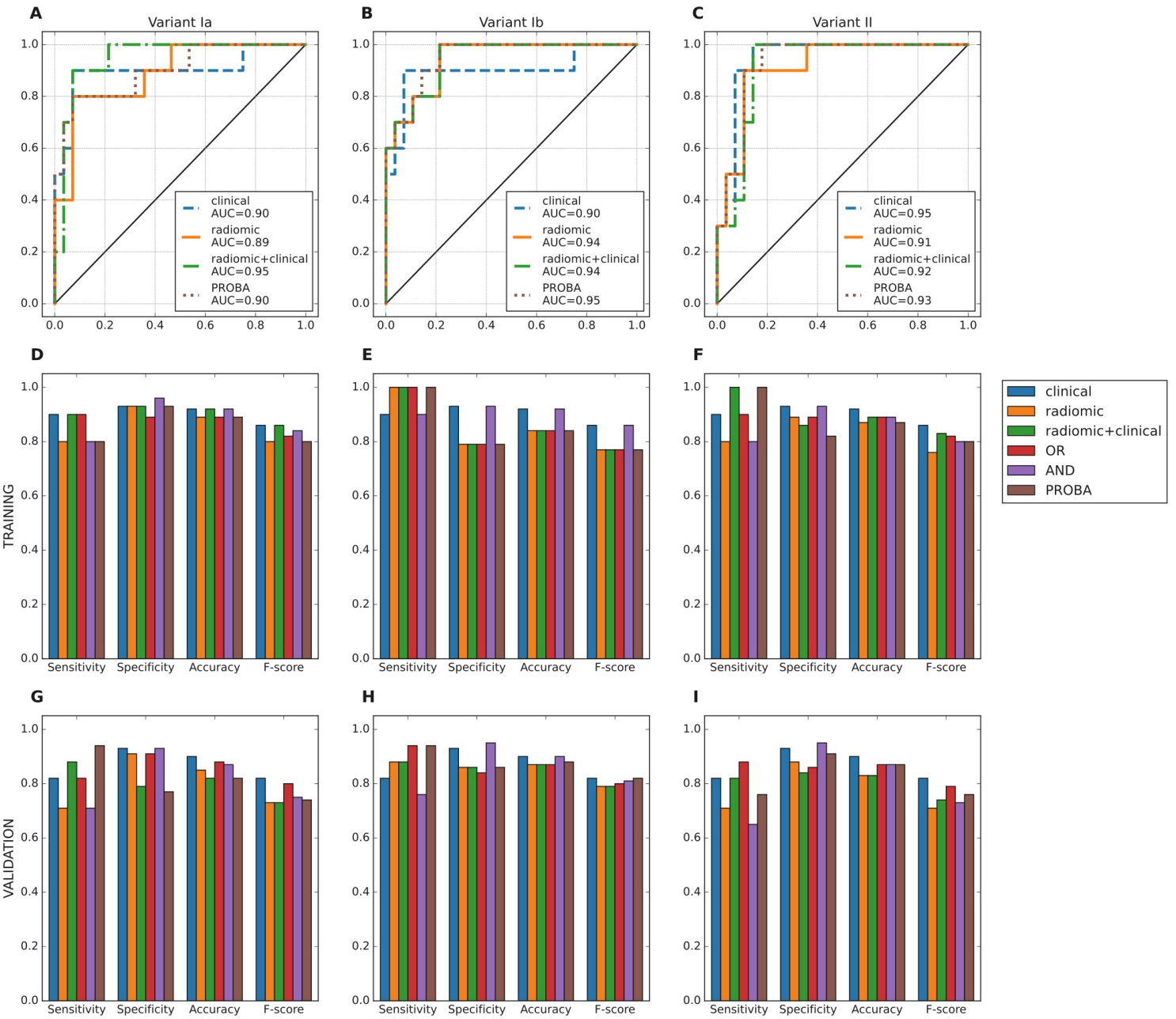

Figure 4. AUC ROC of the selected models (clinical, radiomic, radiomic+clinical) and their ensembles for the training set (A-C). Comparison of statistical measures for the selected model architectures of the considered feature sets and ensembles of those models: training (D-F) and validation (G-I). 

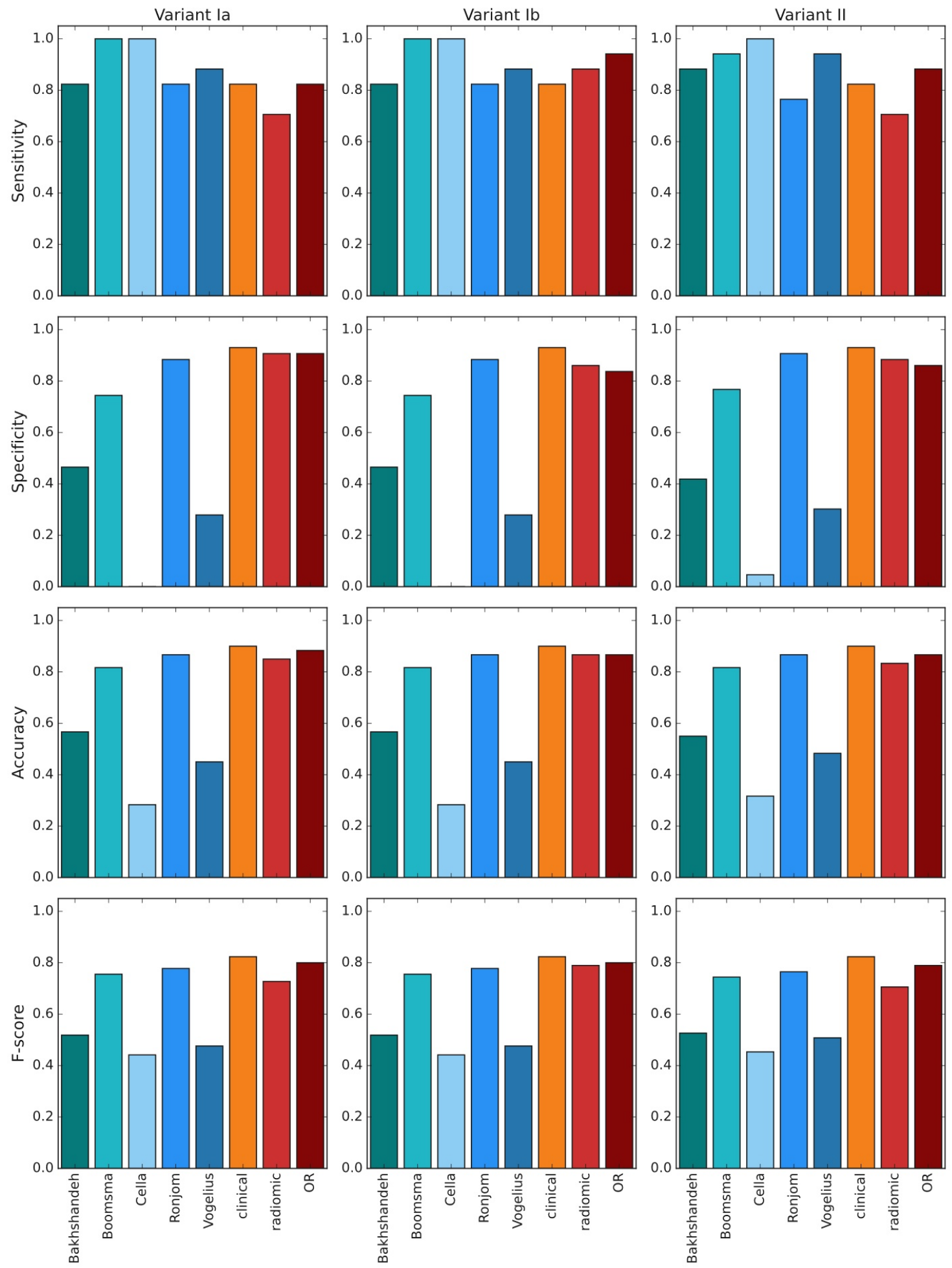

Figure 5. Comparison of quality measures between external NTCP models (cool colors, 5 bars from the left) and our top model selections (warm colors, 3 bars from the right), validation data. 

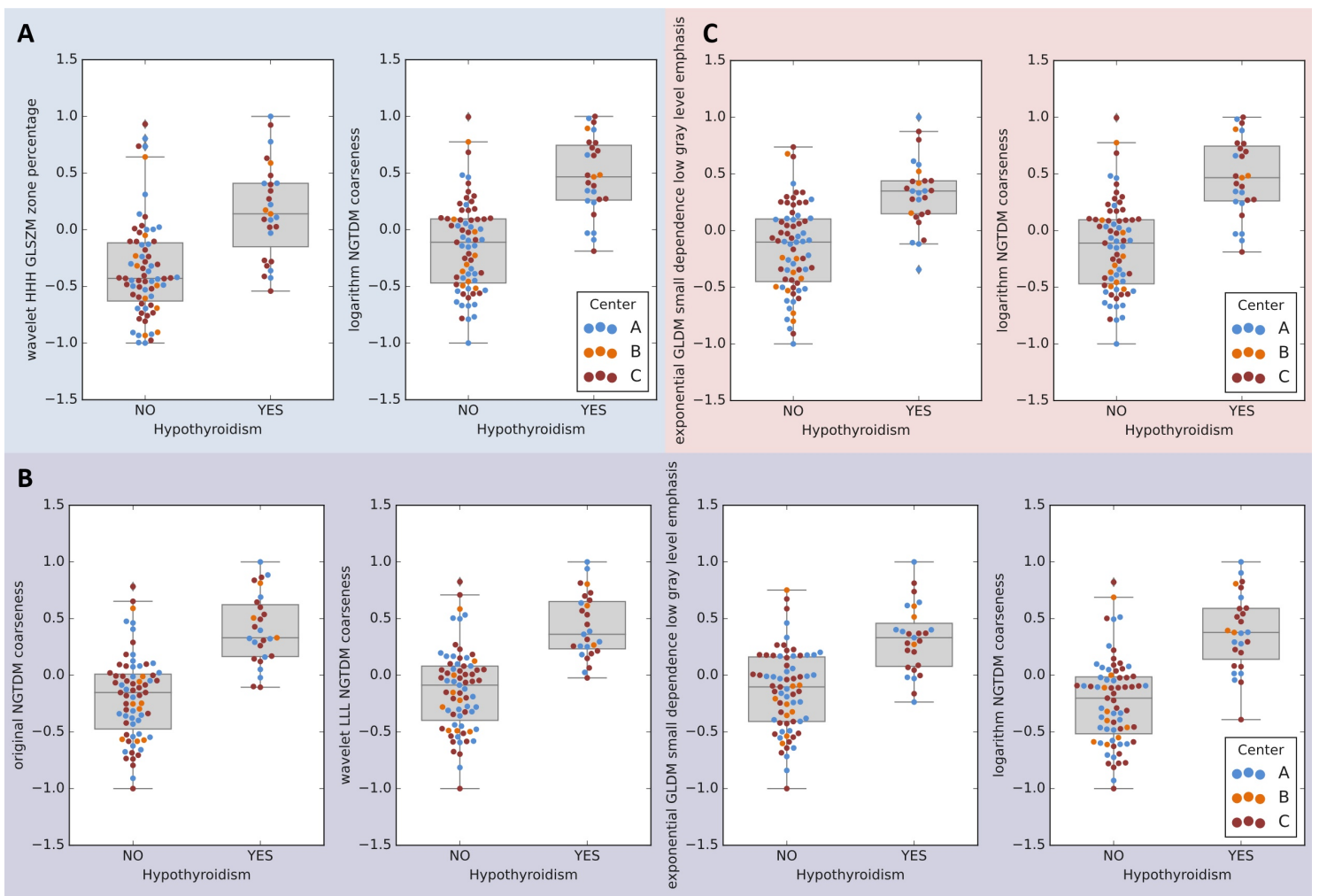

Figure 6. Transformed (normalized and scaled) values of features retained in radiomic models. A: variant Ia, B: variant: $\mathrm{Ib}$, C: variant II.

\section{Discussion}

Here, using data from 98 patients with OPC treated with definite RT, we identified radiomic features predicting the occurrence of RIHT in 2-year follow-up and contrasted our radiomic-based model with published NTCP models based on clinical and dosimetric parameters. Our model performed comparably to models published by Rønjom et al. [8] and by Boomsma et al. [9] and better than three other external models [37-39]. Since predictions based on CT-derived radiomic features are agnostic to dose distribution, they reflect the baseline susceptibility of individual thyroid glands to damage inflicted by radiation doses typical for head and neck cancer RT. They may be therefore leveraged to identify patients in whom close attention should be paid to minimize radiation to the thyroid and the risk of RIHT; alternatively, dosimetric/clinical and radiomic models can be combined to improve prediction accuracy.

Most features in our radiomic model were calculated in filtered CT images and described nonuniformity or coarseness of thyroid region, sometimes emphasizing low gray levels. Invariably higher values of these features, indicating greater non-homogeneity, especially of darker (lower Hounsfield units) regions of thyroid, were characteristic for patients who later developed RIHT.

It must be noted that several articles reported that attenuation and size of an otherwise normal-appearing thyroid might serve as an imaging biomarker for hypothyroidism. Noteworthy, this phenomenon was described for both diagnostic [40] and low dose CT [41]. Approximately $25 \%$ of the body iodine is accumulated in the thyroid gland [42]. This results in hyperdense appearance on the CT scan. Older works postulated that decreased density may be related to disturbances in iodine accumulation and/or processing e.g., inflammation $[43,44]$. Providing biological rationale behind the signal from radiomic features is beyond the scope of our study; however, we can speculate that our radiomic pipeline might detect even more subtle differences in the signal from thyroid gland. Such disturbances may reflect subclinical thyroid insufficiency which makes the gland more susceptible and/or less prone to regeneration. 
Differences in performance between our models and external ones as well as those between different external models likely stem from differences in patient cohort characteristics and chosen treatment protocols, which has already been discussed in detail in a previous study by Nowicka et al. [7] that used data from the same cohort of patients.

An important contribution of our study is analytical pipeline that adds to the recommendations from first reference manual for image biomarker studies [11] and Radiomic Quality Score (RQS) [45]. Addressing criteria from RQS, we reported imaging protocols from participating institutions and verified the stability of features by segmentation perturbation, reducing the number of features and applying multiple comparisons correction. Our simulation study showed that some features are unstable with respect to inaccuracies in thyroid contouring; however, we identified a subset of relatively stable features, corresponding with the results of study on robustness of radiomic features by Zwanenburg et al. [46]. Then, we scaled and normalized the features and performed batch effect correction in one variant of the analysis. This pipeline may be reused and extended for similar projects requiring combining data from many institutions or imaging machines.

We included non-radiomic patient characteristics in the study, reported models' quality statistics and validated models. Patients were recruited prospectively in 3 clinics for the study by Nowicka et al. with the same endpoint of RIHT [7]; however, radiomic analysis was included in the protocol at the later stage. In the place of validation against "golden standard", which is not established for prediction of RIHT, we compared our models with those by other authors. As reported by reviews of radiomic studies [47,48], full adherence to the guidelines is rarely achieved; however even partial compliance improves the quality of research." A TRIPOD guidelines checklist that describes the critical aspects of our work has been provided in the supplementary materials (Table S8).

Our study has several limitations inherent to radiomic studies. The observed batch effects of radiomic features related to oncologic centers (possibly due to use of different CT machines or their settings) may be, for research purposes, solved by batch effect removal tools developed for other high-throughput studies [34,49]. However, such procedure complicates the translation of results and has not been extensively validated in terms of preserving data integrity. Furthermore, our data set has a relatively small sample size with respect to number of features and the developed models require further validation before they can be applied in the clinical setting. Although the validation groups were sufficiently numbered to detect statistically significant differences exceeding $13 \%$ of accuracy it would mandate a larger study to confirm true non-interiority or equivalence of the radiomic and NTCP models, but owing to the presented results planning such an endeavor is possible. Estimating the sample size, if we were to achieve significant difference between AUC of clinical and radiomic models, keeping the current ratio of patient with the complications, we would have to collect data from at least 179 patients who developed RIHT after radiotherapy and from 500 patients, who did not.

An additional benefit of validation in external cohort, ideally from different population, is verification whether the models are not overfitted to our study population. Testing of models developed using data from center A (variant I) on data from center B and $\mathrm{C}$ did not indicate significant overfitting. However, this might be also the result of similarity of studied patient groups that all recruited from Polish population that is quite uniform ethnically.

\section{Conclusions}

Radiomic models reliant on CT scans showed a similar or better ability to predict RIHT in OPC patients compared with the best currently used NTCP models. Additionally, radiomic models are independent from treatment planning and readily deployable across different imaging data.

Supplementary Materials: The following are available online at https: / www.mdpi.com/article / 10.3390/cancers13215584/s1, Figure S1: Normalization and scaling of radiomic features, Figure S2: Batch effect correction, Figure S3: Distribution of radiomic features included in models, Table S1: Raw 
patients' data, Table S2: Raw values of radiomic features, Table S3: Parameters of analyzed models' architectures, Table S4: Stability of radiomic features, Table S5: Univariate analysis and filtration of radiomic features, Table S6: List of radiomic features selected after univariate analysis, Table S7: Quality metrics for all analyzed models, Table S8: Tripod checklist for our study.

Author Contributions: Conceptualization, Z.N., W.F. and B.T.; Data curation, U.S., S.G., Z.N., A.P.-U., R.B., T.L., T.R., J.F. and B.T.; Formal analysis, U.S., S.G. and W.F.; Funding acquisition, Z.N., W.F. and B.T.; Investigation, U.S., S.G., Z.N., A.P.-U., R.B., T.L., T.R., J.F. and B.T.; Methodology, U.S., S.G., Z.N. and B.T.; Project administration, W.F. and B.T.; Resources, A.P.-U., R.B., T.L., T.R., J.F. and B.T.; Software, U.S. and S.G.; Supervision, W.F.; Validation, U.S., S.G. and Z.N.; Visualization, U.S. and S.G.; Writing-original draft, U.S., S.G., Z.N., W.F. and B.T.; Writing—review and editing, U.S., S.G., Z.N., A.P.-U., R.B., T.L., T.R., J.F., W.F. and B.T. All authors have read and agreed to the published version of the manuscript.

Funding: This research was funded by the FIRST TEAM project financed from the Smart Growth Operational Program and coordinated by the Foundation for Polish Science, by the PRELUDIUM project financed by the National Science Center (NCN) (2016/21/N/NZ5/01938) granted to BT, by the Medical University of Lodz grant number 564/1-000-00/564-20-025 granted to Z.N.

Institutional Review Board Statement: The study was conducted according to the guidelines of the Declaration of Helsinki, and approved by the Bioethics Committee of the Medical University of Lodz (KE/7/10, RNN/65/18).

Informed Consent Statement: Informed consent was obtained from all subjects involved in the study.

Data Availability Statement: Raw, anonymized clinical data and values of calculated radiomic features were included in Supplementary Materials.

Acknowledgments: Bartłomiej Tomasik gratefully acknowledges the financial support provided by the Foundation for Polish Science and by the Polish National Agency for Academic Exchange (the Walczak Program).

Conflicts of Interest: The authors declare no conflict of interest.

Abbreviations
$\begin{array}{ll}\text { The following abbreviations are used in this manuscript: } \\ \text { AUC } & \text { area under curve } \\ \text { CT } & \text { computed tomography } \\ \text { FDR } & \text { false discovery rate } \\ \text { GLCM } & \text { gray level cooccurrence matrix } \\ \text { GLDM } & \text { gray level dependence matrix } \\ \text { GLRLM } & \text { gray level run length matrix } \\ \text { GLSZM } & \text { gray level size zone matrix } \\ \text { GPC } & \text { Gaussian process classifier } \\ \text { HNG } & \text { head and neck cancer } \\ \text { ICC } & \text { inter-class correlation coefficient } \\ \text { IMRT } & \text { intensity-modulated radiation therapy } \\ \text { MLP } & \text { multilayer perceptron } \\ \text { NGTDM } & \text { neighborhood gray tone difference matrix } \\ \text { NTCP } & \text { normal tissue complication probability } \\ \text { OAR } & \text { organ at risk } \\ \text { OPC } & \text { oropharyngeal cancer } \\ \text { PACS } & \text { picture archiving and communication system } \\ \text { ROI } & \text { region of interest } \\ \text { RT } & \text { radiation therapy } \\ \text { RIHT } & \text { radiation-induced hypothyroidism }\end{array}$

Appendix A. List of Calculated Radiomic Features

Shape features

- elongation 
- flatness

- least axis length

- major axis length

- maximum 2D diameter column

- maximum 2D diameter row

- maximum 2D diameter slice

- maximum 3D diameter

- mesh volume

- minor axis length

- $\quad$ sphericity

- $\quad$ surface area

- $\quad$ surface volume ratio

- voxel volume

First order features

- 10. percentile

- 90. percentile

- $\quad$ energy

- entropy

- interquartile range

- kurtosis

- maximum

- mean absolute deviation

- mean

- median

- minimum

- range

- robust mean absolute deviation

- $\quad$ root mean squared

- skewness

- $\quad$ total energy

- uniformity

- variance

Gray level cooccurrence matrix-based (GLCM) features

- autocorrelation

- cluster prominence

- cluster shade

- cluster tendency

- contrast

- correlation

- difference average

- difference entropy

- difference variance

- $\quad$ inverse difference (ID), homogeneity 1

- $\quad$ inverse difference moment (IDM), homogeneity 2

- $\quad$ inverse difference moment normalized (IDMN)

- $\quad$ inverse difference normalized (IDN)

- $\quad$ informational measure of correlation 1 (IMC1)

- $\quad$ informational measure of correlation 2 (IMC2)

- inverse variance

- joint average

- joint energy

- joint entropy

- maximal correlation coefficient (MCC) 
- $\quad$ maximum probability

- $\quad$ sum average

- sum entropy

- $\quad$ sum squares

Gray level dependence matrix-based (GLDM) features

- dependence entropy

- dependence nonuniformity

- dependence nonuniformity normalized

- dependence variance

- gray level nonuniformity

- gray level variance

- $\quad$ high gray level emphasis

- large dependence emphasis

- $\quad$ large dependence high gray level emphasis

- large dependence low gray level emphasis

- low gray level emphasis

- $\quad$ small dependence emphasis

- $\quad$ small dependence high gray level emphasis

- $\quad$ small dependence low gray level emphasis

Gray level run length matrix-based (GLRLM) features

- gray level nonuniformity

- gray level nonuniformity normalized

- gray level variance

- $\quad$ high gray level run emphasis

- long run emphasis

- $\quad$ long run high gray level emphasis

- long run low gray level emphasis

- low gray level run emphasis

- run entropy

- $\quad$ run length nonuniformity

- $\quad$ run length nonuniformity normalized

- run percentage

- run variance

- $\quad$ short run emphasis

- $\quad$ short run high gray level emphasis

- $\quad$ short run low gray level emphasis

Gray level size zone matrix-based (GLSZM) features

- gray level nonuniformity

- $\quad$ gray level nonuniformity normalized

- gray level variance

- $\quad$ high gray level zone emphasis

- large area emphasis

- $\quad$ large area high gray level emphasis

- large area low gray level emphasis

- low gray level zone emphasis

- $\quad$ size zone nonuniformity

- $\quad$ size zone nonuniformity normalized

- $\quad$ small area emphasis

- $\quad$ small area high gray level emphasis

- $\quad$ small area low gray level emphasis

- $\quad$ zone entropy

- $\quad$ zone percentage

- zone variance 
Neighborhood gray tone difference matrix-based (NGTDM) features

- busyness

- coarseness

- complexity

- contrast

- strength

\section{References}

1. Brodin, N.P.; Kabarriti, R.; Garg, M.K.; Guha, C.; Tome, W.A. Systematic Review of Normal Tissue Complication Models Relevant to Standard Fractionation Radiation Therapy of the Head and Neck Region Published After the QUANTEC Reports. Int. J. Radiat. Oncol. Biol. Phys. 2018, 100, 391-407. [CrossRef] [PubMed]

2. Vigário, P.; Teixeira, P.; Reuters, V.; Almeida, C.; Maia, M.; Silva, M.; Vaisman, M. Perceived health status of women with overt and subclinical hypothyroidism. Med. Princ. Pract. 2009, 18, 317-322. [CrossRef] [PubMed]

3. Thvilum, M.; Brandt, F.; Almind, D.; Christensen, K.; Hegedüs, L.; Brix, T.H. Excess mortality in patients diagnosed with hypothyroidism: A nationwide cohort study of singletons and twins. J. Clin. Endocrinol. Metab. 2013, 98, 1069-1075. [CrossRef]

4. Hassan, A.; Altamirano-Ufion, A.; Zulfiqar, B.; Boddu, P. Sub-Clinical Hypothyroidism and Its Association With Increased Cardiovascular Mortality: Call for Action. Cardiol. Res. 2017, 8, 31-35. [CrossRef]

5. Rønjom, M.F.; Brink, C.; Bentzen, S.M.; Hegedüs, L.; Overgaard, J.; Johansen, J. Hypothyroidism after primary radiotherapy for head and neck squamous cell carcinoma: Normal tissue complication probability modeling with latent time correction. Radiother. Oncol. 2013, 109, 317-322. [CrossRef] [PubMed]

6. Kamal, M.; Peeler, C.R.; Yepes, P.; Mohamed, A.S.; Blanchard, P.; Frank, S.; Chen, L.; Jethanandani, A.; Kuruvilla, R.; Greiner, B.; et al. Radiation-Induced Hypothyroidism After Radical Intensity Modulated Radiation Therapy for Oropharyngeal Carcinoma. Adv. Radiat. Oncol. 2020, 5, 111-119. [CrossRef] [PubMed]

7. Nowicka, Z.; Tomasik, B.; Papis-Ubych, A.; Bibik, R.; Graczyk, Ł.; Latusek, T.; Rutkowski, T.; Wyka, K.; Fijuth, J.; Schoenfeld, J.D.; et al. Radiation-induced hypothyroidism in patients with oropharyngeal cancer treated with imrt: Independent and external validation of five normal tissue complication probability models. Cancers 2020, 12, 2716. [CrossRef]

8. Rønjom, M.F.; Brink, C.; Bentzen, S.M.; Hegedüs, L.; Overgaard, J.; Petersen, J.B.; Primdahl, H.; Johansen, J. External validation of a normal tissue complication probability model for radiation-induced hypothyroidism in an independent cohort. Acta Oncol. 2015, 54, 1301-1309. [CrossRef]

9. Boomsma, M.J.; Bijl, H.P.; Christianen, M.E.; Beetz, I.; Chouvalova, O.; Steenbakkers, R.J.; Van Der Laan, B.F.; Wolffenbuttel, B.H.; Oosting, S.F.; Schilstra, C.; et al. A prospective cohort study on radiation-induced hypothyroidism: Development of an NTCP model. Int. J. Radiat. Oncol. Biol. Phys. 2012, 84, e351-e356. [CrossRef]

10. Lambin, P.; Rios-Velazquez, E.; Leijenaar, R.; Carvalho, S.; Van Stiphout, R.G.; Granton, P.; Zegers, C.M.; Gillies, R.; Boellard, R.; Dekker, A.; et al. Radiomics: Extracting more information from medical images using advanced feature analysis. Eur. J. Cancer 2012, 48, 441-446. [CrossRef]

11. Zwanenburg, A.; Vallières, M.; Abdalah, M.A.; Aerts, H.J.W.L.; Andrearczyk, V.; Apte, A.; Ashrafinia, S.; Bakas, S.; Beukinga, R.J.; Boellaard, R.; et al. The Image Biomarker Standardization Initiative: Standardized Quantitative Radiomics for High-Throughput Image-based Phenotyping. Radiology 2020, 295, 328-338. [CrossRef]

12. Aerts, H.J.; Velazquez, E.R.; Leijenaar, R.T.; Parmar, C.; Grossmann, P.; Carvalho, S.; Bussink, J.; Monshouwer, R.; Haibe-Kains, B.; Rietveld, D.; et al. Decoding tumour phenotype by noninvasive imaging using a quantitative radiomics approach. Nat. Commun. 2014, 5, 4006. [CrossRef]

13. Leijenaar, R.T.; Carvalho, S.; Hoebers, F.J.; Aerts, H.J.; Van Elmpt, W.J.; Huang, S.H.; Chan, B.; Waldron, J.N.; Osullivan, B.; Lambin, P. External validation of a prognostic CT-based radiomic signature in oropharyngeal squamous cell carcinoma. Acta Oncol. 2015, 54, 1423-1429. [CrossRef] [PubMed]

14. Nardone, V.; Tini, P.; Pastina, P.; Botta, C.; Reginelli, A.; Carbone, S.F.; Giannicola, R.; Calabrese, G.; Tebala, C.; Guida, C.; et al Radiomics predicts survival of patients with advanced non-small cell lung cancer undergoing PD-1 blockade using Nivolumab. Oncol. Lett. 2020, 19, 1559-1566. [CrossRef]

15. Vallières, M.; Kay-Rivest, E.; Perrin, L.J.; Liem, X.; Furstoss, C.; Aerts, H.J.; Khaouam, N.; Nguyen-Tan, P.F.; Wang, C.S.; Sultanem, K.; et al. Radiomics strategies for risk assessment of tumour failure in head-and-neck cancer. Sci. Rep. 2017, 7, 1-14. [CrossRef]

16. Grossmann, P.; Stringfield, O.; El-Hachem, N.; Bui, M.M.; Rios Velazquez, E.; Parmar, C.; Leijenaar, R.T.; Haibe-Kains, B.; Lambin, P.; Gillies, R.J.; et al. Defining the biological basis of radiomic phenotypes in lung cancer. eLife 2017, 6, e23421. [CrossRef]

17. Leijenaar, R.T.; Bogowicz, M.; Jochems, A.; Hoebers, F.J.; Wesseling, F.W.; Huang, S.H.; Chan, B.; Waldron, J.N.; O'Sullivan, B.; Rietveld, D.; et al. Development and validation of a radiomic signature to predict HPV (p16) status from standard CT imaging: A multicenter study. Br. J. Radiol. 2018, 91, 2017049811075. [CrossRef] [PubMed]

18. Cong, M.; Feng, H.; Ren, J.L.; Xu, Q.; Cong, L.; Hou, Z.; Wang, Y.; Shi, G. Development of a predictive radiomics model for lymph node metastases in pre-surgical CT-based stage IA non-small cell lung cancer. Lung Cancer 2020, 139, 73-79. [CrossRef] [PubMed] 
19. Kwan, J.Y.Y.; Su, J.; Huang, S.H.; Ghoraie, L.S.; Xu, W.; Chan, B.; Yip, K.W.; Giuliani, M.; Bayley, A.; Kim, J.; et al. Radiomic Biomarkers to Refine Risk Models for Distant Metastasis in HPV-related Oropharyngeal Carcinoma. Int. J. Radiat. Oncol. Biol. Phys. 2018, 102, 1107-1116. [CrossRef]

20. Sun, R.; Limkin, E.J.; Vakalopoulou, M.; Dercle, L.; Champiat, S.; Han, S.R.; Verlingue, L.; Brandao, D.; Lancia, A.; Ammari, S.; et al. A radiomics approach to assess tumour-infiltrating CD8 cells and response to anti-PD-1 or anti-PD-L1 immunotherapy: An imaging biomarker, retrospective multicohort study. Lancet Oncol. 2018, 19, 1180-1191. [CrossRef]

21. Pérez-Morales, J.; Tunali, I.; Stringfield, O.; Eschrich, S.A.; Balagurunathan, Y.; Gillies, R.J.; Schabath, M.B. Peritumoral and intratumoral radiomic features predict survival outcomes among patients diagnosed in lung cancer screening. Sci. Rep. 2020, 10, 1-15. [CrossRef]

22. Vaidya, P.; Bera, K.; Gupta, A.; Wang, X.; Corredor, G.; Fu, P.; Beig, N.; Prasanna, P.; Patil, P.D.; Velu, P.D.; et al. CT derived radiomic score for predicting the added benefit of adjuvant chemotherapy following surgery in stage I, II resectable non-small cell lung cancer: A retrospective multicohort study for outcome prediction. Lancet Digit. Health 2020, 2, e116-e128. [CrossRef]

23. Coroller, T.P.; Agrawal, V.; Huynh, E.; Narayan, V.; Lee, S.W.; Mak, R.H.; Aerts, H.J. Radiomic-Based Pathological Response Prediction from Primary Tumors and Lymph Nodes in NSCLC. J. Thorac. Oncol. 2017, 12, 467-476. [CrossRef]

24. Sha, X.; Gong, G.; Qiu, Q.; Duan, J.; Li, D.; Yin, Y. Discrimination of mediastinal metastatic lymph nodes in NSCLC based on radiomic features in different phases of CT imaging. BMC Med. Imaging 2020, 20, 1-8. [CrossRef] [PubMed]

25. Van Dijk, L.V.; Brouwer, C.L.; van der Schaaf, A.; Burgerhof, J.G.; Beukinga, R.J.; Langendijk, J.A.; Sijtsema, N.M.; Steenbakkers, R.J. CT image biomarkers to improve patient-specific prediction of radiation-induced xerostomia and sticky saliva. Radiother. Oncol. 2017, 122, 185-191. [CrossRef]

26. Gabryś, H.S.; Buettner, F.; Sterzing, F.; Hauswald, H.; Bangert, M. Design and Selection of Machine Learning Methods Using Radiomics and Dosiomics for Normal Tissue Complication Probability Modeling of Xerostomia. Front. Oncol. $2018,8,35$. [CrossRef]

27. Krafft, S.P.; Rao, A.; Stingo, F.; Briere, T.M.; Court, L.E.; Liao, Z.; Martel, M.K. The utility of quantitative CT radiomics features for improved prediction of radiation pneumonitis. Med. Phys. 2018, 45, 5317-5324. [CrossRef] [PubMed]

28. Lucia, F.; Bourbonne, V.; Visvikis, D.; Miranda, O.; Gujral, D.M.; Gouders, D.; Dissaux, G.; Pradier, O.; Tixier, F.; Jaouen, V.; et al. Radiomics Analysis of 3D Dose Distributions to Predict Toxicity of Radiotherapy for Cervical Cancer. J. Pers. Med. 2021, 11, 398. [CrossRef]

29. Rossi, L.; Bijman, R.; Schillemans, W.; Aluwini, S.; Cavedon, C.; Witte, M.; Incrocci, L.; Heijmen, B. Texture analysis of 3D dose distributions for predictive modelling of toxicity rates in radiotherapy. Radiother. Oncol. 2018, 129, 548-553. [CrossRef]

30. Bourbonne, V.; Da-ano, R.; Jaouen, V.; Lucia, F.; Dissaux, G.; Bert, J.; Pradier, O.; Visvikis, D.; Hatt, M.; Schick, U. Radiomics analysis of 3D dose distributions to predict toxicity of radiotherapy for lung cancer. Radiother. Oncol. 2021, 155, 144-150. [CrossRef]

31. Van Griethuysen, J.J.; Fedorov, A.; Parmar, C.; Hosny, A.; Aucoin, N.; Narayan, V.; Beets-Tan, R.G.; Fillion-Robin, J.C.; Pieper, S.; Aerts, H.J. Computational radiomics system to decode the radiographic phenotype. Cancer Res. 2017, 77, e104-e107. [CrossRef]

32. Phil, T. Sikerdebaard/Dcmrtstruct2nii: v1.0.19. 2020. Available online: https://github.com/Sikerdebaard/dcmrtstruct2nii (accessed on 20 August 2021).

33. Yeo, I.K.; Johnson, R. A new family of power transformations to improve normality or symmetry. Biometrika 2000, 87, 954-959. [CrossRef]

34. Johnson, W.E.; Li, C.; Rabinovic, A. Adjusting batch effects in microarray expression data using empirical Bayes methods. Biostatistics 2007, 8, 118-127. [CrossRef]

35. Pedregosa, F.; Varoquaux, G.; Gramfort, A.; Michel, V.; Thirion, B.; Grisel, O.; Blondel, M.; Prettenhofer, P.; Weiss, R.; Dubourg, V.; et al. Scikit-learn: Machine Learning in Python. J. Mach. Learn. Res. 2011, 12, 2825-2830.

36. Raschka, S. MLxtend: Providing machine learning and data science utilities and extensions to Python's scientific computing stack. J. Open Source Softw. 2018, 3, 638. [CrossRef]

37. Bakhshandeh, M.; Hashemi, B.; Mahdavi, S.R.M.; Nikoofar, A.; Vasheghani, M.; Kazemnejad, A. Normal tissue complication probability modeling of radiation-induced hypothyroidism after head-and-neck radiation therapy. Int. J. Radiat. Oncol. Biol. Phys. 2013, 85, 514-521. [CrossRef] [PubMed]

38. Cella, L.; Liuzzi, R.; Conson, M.; D’Avino, V.; Salvatore, M.; Pacelli, R. Development of multivariate NTCP models for radiationinduced hypothyroidism: A comparative analysis. Radiat. Oncol. 2012, 7, 224. [CrossRef]

39. Vogelius, I.R.; Bentzen, S.M.; Maraldo, M.V.; Petersen, P.M.; Specht, L. Risk factors for radiation-induced hypothyroidism: A literature-based meta-analysis. Cancer 2011, 117, 5250-5260. [CrossRef]

40. Maldjian, P.D.; Chen, T. Is visual assessment of thyroid attenuation on unenhanced CT of the chest useful for detecting hypothyroidism? Clin. Radiol. 2016, 71, 1199.e9-1199.e14. [CrossRef]

41. Watane, G.V.; Hammer, M.M. Imaging Biomarkers of Hypothyroidism on Lung Cancer Screening CT. Curr. Probl. Diagn. Radiol. 2021, 50, 807-810. [CrossRef]

42. Cavalieri, R.R. Iodine metabolism and thyroid physiology: Current concepts. Thyroid. Off. J. Am. Thyroid. Assoc. 1997, 7, 177-181. [CrossRef] [PubMed]

43. Arger, P.H.; Jennings, A.S.; Gordon, L.F.; Coleman, B.G.; Axel, L.; Kressel, H.Y.; Baron, R.L. Computed tomography findings in clinically normal and abnormal thyroid patients. J. Comput. Tomogr. 1985, 9, 111-117. [CrossRef] 
44. Kamijo, K. Clinical studies on thyroid CT number in chronic thyroiditis. Endocr. J. 1994, 41, 19-23. [CrossRef]

45. Lambin, P.; Leijenaar, R.T.H.; Deist, T.M.; Peerlings, J. Radiomics: The bridge between medical imaging and personalized medicine. Nat. Rev. Clin. Oncol. 2017, 14, 749-762. [CrossRef] [PubMed]

46. Zwanenburg, A.; Leger, S.; Agolli, L.; Pilz, K.; Troost, E.G.; Richter, C.; Löck, S. Assessing robustness of radiomic features by image perturbation. Sci. Rep. 2019, 9, 1-31. [CrossRef]

47. Sanduleanu, S.; Woodruff, H.C.; de Jong, E.E.; van Timmeren, J.E.; Jochems, A.; Dubois, L.; Lambin, P. Tracking tumor biology with radiomics: A systematic review utilizing a radiomics quality score. Radiother. Oncol. 2018, 127, 349-360. [CrossRef] [PubMed]

48. Park, J.E.; Kim, D.; Kim, H.S.; Park, S.Y.; Kim, J.Y.; Cho, S.J.; Shin, J.H.; Kim, J.H. Quality of science and reporting of radiomics in oncologic studies: room for improvement according to radiomics quality score and TRIPOD statement. Eur. Radiol. 2020, 30, 523-536. [CrossRef] [PubMed]

49. Vuong, D.; Bogowicz, M.; Denzler, S.; Oliveira, C.; Foerster, R.; Amstutz, F.; Gabryś, H.S.; Unkelbach, J.; Hillinger, S.; Thierstein, S.; et al. Comparison of robust to standardized CT radiomics models to predict overall survival for non-small cell lung cancer patients. Med. Phys. 2020, 47, 4045-4053. [CrossRef] 